\title{
Renal Allograft Compartment Syndrome: Is It Possible to Prevent?
}

\author{
G. Damiano ${ }^{a, \star}$, C. Maione ${ }^{a}$, A. Maffongelli $^{a}$, S. Ficarella ${ }^{a}$, L. Carmina ${ }^{a}$, S. Buscemi ${ }^{a}$, V.D. Palumbo $^{a}$, \\ S. De Luca ${ }^{a}$, G. Spinelli ${ }^{a}$, A.I. Lo Monte ${ }^{a}$, and G. Buscemi ${ }^{a, b}$

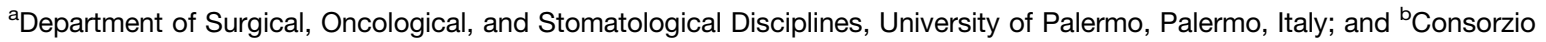 \\ Interuniversitario dei Trapianti d'Organo, Roma, Italy
}

\begin{abstract}
Renal allograft compartment syndrome (RACS) is a complication characterized by increased pressure over 15 to $20 \mathrm{~mm} \mathrm{Hg}$ of the iliac fossa site of transplanted kidney that can lead to a reduction of the blood supply to the graft, resulting in organ ischemia. This study aims to evaluate, through a review of the literature, the incidence, detection, treatment, and possible prevention of RACS. The incidence of this complication, which appears generally in the immediate post-transplantation period, is currently approximately $1 \%$ to $2 \%$ and is underestimated because of poor nosography for the presence of symptoms common to other post-transplantation complications. Doppler ultrasound is indispensable to evaluate the graft function in the immediate postoperative period and in the following days. The onset of RACS involves a surgical decompression of the graft and the subsequent closure of the abdominal wall with tension-free technique. Several authors agree that only the immediate surgical decompression following an early diagnosis can ensure a recovery of the graft. Early detection of the RACS is the key to preventing the loss of the graft. It is desirable to prevent this syndrome by reducing the discrepancy in weight between donor and recipient by $17 \%$. However the shortage of organs makes such a selection not easy; therefore, in cases at risk for RACS, a close instrumental and clinical monitoring of the patient during post-transplantation recovery is recommended, so a prompt surgical decompression can be performed if RACS is suspected.
\end{abstract}

$\mathbf{R}$ ENAL ALLOGRAFT COMPARTMENT SYNDROME (RACS) is an early complication following kidney transplantation. In the absence of prompt treatment, it can have devastating effects, including the loss of the graft. If the transplanted kidney is too big for the size of the shallow false pelvis and the limited retroperitoneal space, increased pressure will be transmitted to the graft and causing graft ischemia with reduction of renal function (by $25 \%$ with an increased pressure between 15 and $20 \mathrm{~mm} \mathrm{Hg}$, and the onset of anuria when the pressure is higher than $20 \mathrm{~mm} \mathrm{Hg}$ ) [1].

The identification of RACS as a clinical entity is very recent [2]; the diagnosis was made for the first time thanks to the definition of abdominal compartment syndrome (ACS), a life-threatening condition characterized by a rapid and sudden increase of intra-abdominal pressure that may arise from abdominal trauma (blunt or penetrating) or non-traumatic conditions such as septic peritonitis, intestinal obstruction/perforation, acute pancreatitis, acute

$0041-1345 / 16$

http://dx.doi.org/10.1016/j.transproceed.2015.12.051 cholecystitis, aortic aneurysm rupture, mesenteric infarction, and organ transplantation. The increase in intraabdominal pressure due to cytokine release, formation of oxygen free radicals, decreased cellular energy production, edema, hypoperfusion, and ischemia can cause rapid deterioration of clinical condition up to multiorgan failure [3,4].

The identification of RACS was observed in renal transplant recipients who had a sudden reduction and/or loss of function of the graft not attributable to either pharmacological or immunological complications [5-8].

The World Society of Abdominal Compartment Syndrome (WSACS) released a classification for intra-abdominal hypertension [9] (Table 1). ACS is defined as a sustained intra-abdominal pression greater than $20 \mathrm{~mm} \mathrm{Hg}$ that is

*Address correspondence to Giuseppe Damiano, University of Palermo, 129 Via del Vespro, 90127, Palermo, Italy. E-mail: giuse. damiano@gmail.com

(c) 2016 by Elsevier Inc. All rights reserved. 360 Park Avenue South, New York, NY 10010-1710 
Table 1. The World Society of Abdominal Compartment Syndrome Grade Classification for Intra-abdominal Hypertension

\begin{tabular}{lc}
\hline $\begin{array}{c}\text { Intra-abdominal } \\
\text { hypertension }\end{array}$ & $\begin{array}{c}\text { Prolonged or repeated } \\
\text { elevation of IAP }>12 \mathrm{~mm} \mathrm{Hg}\end{array}$ \\
Grade I & $12-15 \mathrm{~mm} \mathrm{Hg}$ \\
Grade II & $16-20 \mathrm{~mm} \mathrm{Hg}$ \\
Grade III & $21-25 \mathrm{~mm} \mathrm{Hg}$ \\
Grade IV & $>25 \mathrm{~mm} \mathrm{Hg}$ \\
\hline
\end{tabular}

associated with new organ dysfunction/failure. In consideration of a possible increase of intra-abdominal pressure $>20 \mathrm{~mm} \mathrm{Hg}$, abdominal compartment syndrome should be suspected in order to implement therapeutic methods to timely prevent or cure the onset of complications that would lead to the loss of the graft in transplant recipients.

The recognition of this syndrome in recent years as clinical condition has allowed the recovery of transplanted kidneys that in the past had been lost and for which clinical deterioration was mistakenly attributed to immunological, pharmacological, and/or iatrogenic causes.

\section{Clinical Presentation of RACS}

RACS immediately post-transplantation, which was described for the first time by Humar et al in 1996 [2], has an incidence of $1 \%$ to $2 \%$. However, the data still may be underestimated because of misunderstanding regarding this syndrome; in fact, the symptoms are common to other complications after transplantation (acute tubular necrosis, hyperacute rejection, acute rejection, thrombosis of renal vessels). Commonly, the patient presents with general discomfort, tense abdomen, tachypnea, oliguria, and hypotension [10-13]. There are no characteristic clinical signs indicative of abdominal compartment syndrome. It is assumed that the raised pressure exerted on the transplanted kidney increases the intracapsular pressure, vascular resistance, and the inversion of the wave diastolic flow in the renal artery, thus reducing the blood supply to the graft, with the consequent reduction or loss of function. This hypothesis was supported by experimental studies carried out [14] on transplanted mice by Herrler et al, in which the ischemia-reperfusion injury that raises of intracapsular pressure and decreases renal flow and tubular function can be resolved through a polar capsulotomy of the kidney [15].

\section{Identification of RACS}

Early diagnosis is a critical step to avoid the loss of the graft in the immediate post-transplantation period. Nowadays the identification of patients at risk of RACS is still difficult. However, some progress has been made since some categories of people have been identified who may be considered at higher risk of developing the syndrome because of the significant discrepancy in weight compared to the donors (the pediatric recipients of adult donors) $[12,16]$. Also at higher risk are patients undergoing closure of the muscular wall of the abdomen with a non-tension-sparing technique [13]. In fact, in a study of 538 patients, Ortiz et al have found as statistically significant risk factors for the development of RACS the length and the width of the transplanted kidney with an odd ratio of 1.61 and 2.32, respectively, whereas the body mass index of recipient, vascular anomalies, the incision site, and the site of placement of the graft were not statistically significant [17]. Finally, being that RACS is a variant of the ACS, conditions such as lymphocele, ascites, the preoperative intestinal edema associated with hypoalbuminemia, and the quantity of liquid introduced during anesthesia may be considered other risk factors $[18,19]$.

To date there is no standardized protocol for diagnosing RACS: some authors have proposed measuring intraoperative intra-bladder pressure in pediatric recipients to indirectly estimate intra-abdominal pressure [10]. Several studies point to the role of a Doppler ultrasound (US) of the renal vessels $[15,20-22]$ to identify possible impairment of blood flow, not only in the immediate postoperative period but also in the days that follow. Thanks to the fact that this control is easy to use, inexpensive, noninvasive, and repeatable, it is today the most widely used diagnostic method for monitoring the transplanted kidney. Doppler US scan identifies all those alterations of flow that can be connected with a probable onset of RACS. However, if the reversal of diastolic flow in the intrarenal arteries occurs or there is an absence of flow in renal veins, this may indicate the onset of abdominal compartment syndrome, or other conditions such as acute tubular necrosis or vascular renal thrombosis. In a study of 575 transplanted patients, Horrow et al [20] analyzed the main US vascular abnormalities in the immediate posttransplantation period: the anomalies considered were the lack of flow in the artery and/or renal vein, high peak systolic velocity (with values $>300 \mathrm{~cm} / \mathrm{s}$ ), parvus tardus pulse waveforms, and the marked reduction or absence of parenchymal renal flow. In the aforementioned study 47 patients reported at least 1 of these vascular changes and the Doppler US scan in 16 patients has indicated surgery for compartment syndrome $(n=7)$, renal vein thrombosis $(n=3)$, renal artery thrombosis $(\mathrm{n}=1)$, renal artery and vein thrombosis $(\mathrm{n}=2)$, and vascular steal $(n=1)$. In 2 patients there were no vascular abnormalities at the time of reintervention. In all 16 patients marked reduction of renal parenchymal flow, changes in peak systolic velocity, and renal vascular flow alterations were observed.

The study pointed out that in the immediate postoperative period, a number of vascular changes identified with Doppler examination, in particular including the massive reduction of renal parenchymal flow, put the indication for resurgery quickly to prevent the loss of the graft. However, as already pointed out, this examination is not able to make a differential diagnosis between various causes responsible for the vascular changes before the patient reaches the operating table. Therefore, if the onset of RACS is suspected or possible, the unique opportunity to safeguard the function of the transplanted kidney is to perform a surgical decompression as early as possible by adopting tension-free closure techniques. 


\section{Treatment of RACS}

According to the data of literature examined, there are critical points in terms of both early diagnosis and regarding the timing of the surgical treatment of RACS. The recovery of the transplanted kidney is possible by surgical decompression, which restores the physiological vascular flow.

In the various studies examined, the definitive diagnosis of RACS was made on the operating table, despite the use of Doppler US scan. The flow alterations detected by US are not specific, being common in other post-transplantation complications. In fact, according to Horrow et al [20], Doppler US monitoring of transplanted kidney located approximately $8 \%$ of patients with abnormal flow; of these, only $34 \%$ were subjected to an urgent reintervention, and half of these were really suffering from RACS for which decompression warranted restoration of function. Given this experience with patients affected by RACS, there are experiences of other authors who acted before the onset of RACS, performing surgical decompression techniques during the transplantation, as in studies of Richards [23] and Pentlow [24]. In a series of 600 transplanted patients, Wood [13] performed decompression techniques on 134 (22\%); among these, $123(20.5 \%)$ underwent closure of the abdominal wall with polypropylene prosthesis during transplantation, as the discrepancy between the space of positioning and the size of the kidney was at high risk of onset of RACS in the immediate postoperative period; in 11 patients $(1.8 \%)$ decompression was performed early posttransplantation for the onset of a real RACS. In both cases the recovery, survival of kidney and patients were almost identical. Therefore, the only data in common in all the studies examined is the surgical technique, which consists of the creation of a space of greater dimensions in which to allocate the kidney to reduce the endoabdominal pressure through the use of prosthesis. For this purpose, based on the experience of the various groups, the following different types of prostheses were used [25]: (1) porcine mesh "Permacol"; (2) PTFE dual mesh prosthesis; and (3) polypropyleneassisted mesh hood fascial closure (MHFC).

Richards et al [23] have used prosthetic porcine collagen (Permacol) to facilitate the closure of the abdominal wall after transplantation of a kidney from adult cadaver donor to a 2-year-old recipient who weighed $12 \mathrm{~kg}$. The prosthesis in this case was implanted between the rectus muscles and fixed with continuous suture in PDS, without complications. Subsequently, the authors treated two other pediatric patients the same way.

Similarly, Pentlow et al [24] published a study on the use of porcine prostheses in the closure of the abdominal wall in 5 pediatric recipients (5-12 years) of adult donors, followed for 3 years, during which it proved to be a useful aid in all cases of kidney transplantation in which there was a size discrepancy between donor and recipient.

Our group used prosthetics dual-mesh PTFE, for the onset of RACS in a 42-year-old female kidney transplanted patient, in which compartment syndrome was due to the narrow space in which the graft was transplanted [1]. The closure of the abdominal wall with tension-free technique has allowed us to recover the functionality of the graft.

Other authors as Nguan et al [26] and Wood [13] described the use of MHFC for closure of the abdominal wall with tension-free technique both during kidney transplantation in patients considered at risk of RACS is in the post-transplantation period after verification of the increase in intra-abdominal pressure.

In further studies examined, if the onset of RACS is expected and it is not possible to decompress the abdominal wall using the techniques above described due to a high risk of infection, surgical techniques have been implemented that involve the allocation of the transplanted kidney in the subcutaneous or intraperitoneal site. Ball et al [11] adopted the subcutaneous positioning of transplanted kidney in 3 patients diagnosed with RACS, transplanted by transretroperitoneal way in the right iliac fossa. In 2 patients there was a significant difference in size compared to donors. The follow up of these cases showed an excellent renal function after transplantation. Koss et al [27] describes the intraperitoneal positioning of previously transplanted kidney in the iliac fossa that caused a compartment syndrome due to excessive intraabdominal tension. Ball et al [11] reported intraperitoneal placement of the transplanted kidney as treatment of RACS in 8 patients. In both studies, there were no surgical complications and there was a restoration of function of the graft after the reintervention.

\section{CONCLUSION}

Early detection of RACS is the key to preventing the loss of the graft, to facilitate surgical decompression that will allow functional recovery of the transplanted kidney.

To date the only means to identify the syndrome are clinical suspicion and monitoring by Doppler US, which, however, may provide morphofunctional information common to several post-transplantation complications, instead of allowing an early certain diagnosis of RACS. Benefits were shown in a study by Fontana et al on pediatric recipients using intravesical pressure as an indirect estimation of intra-abdominal pressure. In the same study, color Doppler was used both in the immediate postoperative period to evaluate vascular changes consistent with the development of a compartment syndrome after surgery and to determine surgical timing. The reoperation in the immediate post-transplantation period appears to be burdened by a significant risk for both for the patient and the graft. Indeed, it is necessary to identify all the conditions and factors already present before transplantation that could raise suspicion of a development of RACS and act preventively before the onset of this syndrome. Ideally, the allocation of organs would follow a discrepancy in weight between donor and recipient less than $17 \%$; however, due to the shortage of organs, this criterion is not always possible. Therefore in our opinion, it is necessary to identify groups at risk of developing RACS before being transplanted and 
implementing methods of abdominal decompression during the transplantation itself.

Based on studies of literature we reviewed and our experience we have identified the following risk conditions:

(1) anatomical conditions of the recipient:

- Small basin.

- Polycystic kidneys in situ.

- All those conditions in which difficulty of allocation of kidney in the iliac fossa is expected.

(2) Conditions related to the graft:

- Discrepancy between donor weight and weight of the recipient.

- Organs from obese donor.

- Organs from adult donors for pediatric recipients.

(3) Iatrogenic conditions post-transplantation:

- Closure of the abdominal wall with excessive tension.

- Massive post-transplantation lymphoceles.

Lacking guidelines universally accepted in the various transplant centers, the closure of the muscle-aponeurotic wall with tension-free techniques should be adopted for all of these conditions described above to prevent RACS. Furthermore, there are no guidelines regarding the use of prostheses; in general a biological prosthesis is used (Permacol) when the use of synthetic prostheses is not favorable or is contraindicated, as in the case of a potential source of infection. However, synthetic prostheses are currently used [28] and in the studies reviewed there were differences in the incidence of infection and/or complications. Indeed, we must dispel the subjective opinion entry of the increased incidence of infectious complications by using prosthetic mesh in such immunosuppressed patients.

Finally, in those rare cases in which the closure of the wall by affixing the prosthetic mesh is not favorable, or is contraindicated, there are alternative techniques such as positioning the graft in the subcutaneous or intraperitoneal space.

\section{REFERENCES}

[1] Maione C, Gambino G, Di Bona A, Luna E, Turco D, Scio A, et al. PTFE mesh in renal allograft compartment syndrome. Transplant Proc 2006;38:1049-50.

[2] Humar A, Sharpe J, Hollomby D. Salvage of a renal allograft with renal vein occlusion secondary to extrinsic compression. Am J Kidney Dis 1996;28:622-3.

[3] Bendahan J, Coetzee CJ, Papagianopoulos C, et al. Abdominal compartment syndrome. J Trauma 1995;38:152-3.

[4] Nebelkopf H. Abdominal compartment syndrome. Am J Nurs 1999;99:53-6. 58, 60.

[5] De Cleva R, Silva FP, Zilberstein B, Machado DJ. Acute renal failure due to abdominal compartment syndrome: report on four cases and literature review. Rev Hosp Clin Fac Med S Paulo 2001;56:123-30.

[6] Berger P, Nijsten MW, Paling JC, Zwaveling JH. The abdominal compartment syndrome: a complication with many faces. Neth J Med 2001;58:197-203.

[7] Doty JM, Saggi BH, Blocher CR, Fakhry I, Gehr T, Sica D, et al. Effects of increased renal parenchymal pressure on renal function. J Trauma 2000;48:874-7.

[8] De Waele JJ, De Laet I. Intra-abdominal hypertension and the effect on renal function. Acta Clin Belg Suppl 2007;2:371-4.
[9] Malbrain ML, Cheatham ML, Kirkpatrick A, Sugrue M, Parr M, De Waele J, et al. Results from the International Conference of Experts on Intra-Abdominal hypertension and Abdominal Compartment Syndrome. I. Definitions. Intensive Care Medicine 2006;32:1722-32.

[10] Fontana I, Bertocchi M, Centanaro M, Varotti G, Santori G, Mondello R, et al. Abdominal compartment syndrome: an underrated complication in pediatric kidney transplantation. Transplant Proc 2014;46:2251-3.

[11] Ball CG, Kirkpatrick AW, Yilmaz S, Monroy M, Nicolaou S, Salazar A. Renal allograft compartment syndrome: an underappreciated postoperative complication. Am J Surg 2006;191: 619-24.

[12] Beasley KA, McAlister VC, Luke PP. Mesh hood fascial closure in renal allograft compartment syndrome. Transplant Proc 2003;35:2418-9.

[13] Wood LN, Yang W, Annamalai A. Mesh hood fascial closure is a safe alternative to prevent renal allograft compartment syndrome during kidney transplantation. Transplant Proc 2015;47: 1845-9.

[14] Herrler T, Tischer A, Meyer A, Feiler S, Guba M, Nowak S, et al. The intrinsic renal compartment syndrome: new perspectives in kidney transplantation. Transplantation 2010;89:40-6.

[15] Heer MK, Trevillian PR, Hardy DB, Hibberd AD. Salvaging kidneys with renal allograft compartment syndrome. Transpl Int 2012;25:e47-9.

[16] Khemchandani SI. Mesh hood fascial closure in renal allograft compartment syndrome in pediatric transplantation. Urol J 2014;11:1335-7.

[17] Ortiz J, Parsikia A, Horrow MM, Khanmoradi K, Campos S, Zaki R. Risk factors for renal allograft compartment syndrome. Int Surg 2014;99:851-6.

[18] Kirkpatrick AW, Balogh Z, Ball CG, Ahmed N, Chun R, McBeth P, et al. The secondary abdominal compartment syndrome: iatrogenic or unavoidable? J Am Coll Surg 2006;202:668-79.

[19] Fontana I, Bertocchi M, Centanaro M, Diviacco P, De Negri A, Ghinolfi D, et al. Renal transplant compartment syndrome: a case report. Transplant Proc 2008;40:2065-6.

[20] Horrow MM, Parsikia A, Zaki R, Ortiz J. Immediate postoperative sonography of renal transplants: vascular findings and outcomes. AJR Am J Roentgenol 2013;201:W479-86.

[21] Thiyagarajan UM, Bagul A, Mohamed I, Nicholson ML. Post-biopsy renal allograft compartment syndrome: Addressing the problem, illustrated with a case report. Int J Surg Case Rep 2011;2: $188-90$.

[22] Wiebe S, Kellenberger CJ, Khoury A, Miller SF. Early Doppler changes in a renal transplant patient secondary to abdominal compartment syndrome. Pediatr Radiol 2004;34:432-4.

[23] Richards SK, Lear PA, Huskisson L, Saleem MA, Morgan JD. Porcine dermal collagen graft in pediatric renal transplantation. Pediatr Transplant 2005;9:627-9.

[24] Pentlow A, Smart NJ, Richards SK, Inward CD, Morgan JD. The use of porcine dermal collagen implants in assisting abdominal wall closure of pediatric renal transplant recipients with donor size discrepancy. Pediatr Transplant 2008;12: 20-3.

[25] Palumbo VD, Damiano G, Gioviale MC, Lo Monte AI. Mesh or no mesh: a hamletic dilemma to prevent renal allograft compartment syndrome (RACS). Ann Ital Chir 2014;85:282-6.

[26] Nguan CY, Beasley KA, McAlister VC, Luke PP. Treatment of renal transplant complications with a mesh hood fascial closure technique. Am J Surg 2007;193:119-21.

[27] Koss WG, Johnson LB, Kuo PC. Retroperitoneal compartment syndrome after renal transplantation. Am Surg 2000;66:80-1.

[28] Lo Monte AI, Damiano G, Maione C, Gioviale MC, Lombardo C, Buscemi G, et al. Use of intraperitoneal ePTFE Gore dual-mesh plus in a giant incisional hernia after kidney transplantation: a case report. Transplant Proc 2009;41(4):1398-401. 